\title{
EFEITOS DE MISTURAS DE BENTAZON E PARAQUAT NO CONTROLE DE PLANTAS DANINHAS E NA CULTURA DO FEIJÃO (Phaseolus vulgaris L.) (NOTA PRÉVIA)
}

B.N. RODRIGUES* \& R. VICTORIA FILHO**

* Pesquisador do Instituto Agronômico do Paraná - IAPAR - Londrina - PR.

* Professor Assistente Doutor do Departamento de Agricultura e Horticultura - ESALQ/USP Piracicaba - SP

\section{RESUMO}

O objetivo da presente pesquisa foi estudar a seletividade da mistura bentazon (3-isopropil-2,1, 3benzothiodiazinona-(4)-2,2-dióxido) com paraquat (1,1'-dimetil-4,4'-bipiridilio dicloreto) para as cultivares de feijão 'Carioca' e 'Moruna'; alêm do efeito dessa mistura no controle de algumas plantas daninhas e possiveis efeitos sinergisticos resultantes das diferentes misturas. O experimento está sendo conduzido em condições de campo na área experimental do Departamento de Agricultura e Horticultura da ESALQ em Piracicaba-SP, como tambêm em casa de vegetação. O delineamento experimental foi o de blocos ao acaso com quatro repetições. O critério de avaliação adotado foi o de "percentagem de injúria", às cultivares e às espécies de plantas daninhas, através de avaliação visual. A verificacão de sinergismo foi feita utilizando-se a fórmula de Gowing citada por Colby (1).

De acordo com esse método, verificou -se que, para ambas as cultivares de feijão, a injúria observada foi menor que a esperada, o que caracterizou um evidente antagonismo entre as misturas desses herbicidas, em relação à cultura. Em relação ao capim-colchão (Digitaria sanquinalis (L.) Scop.) todas as misturas ensaiadas foram também antagonísticas. O sinergismo entre esses produtos ficou bastante caracterizado no controle da guanxuma (Sida glaziovii K. Sch.) e beldroega (Portulacca oleracea L.) principalmente nas doses de $0,48+0,05 \mathrm{~kg} / \mathrm{ha}$ de bentazon + paraquat, respectivamente, notadamente ao $6 .^{\circ}$ e $8 .^{\circ}$ dia após a aplicação. Quanto ao fedegoso (Cassia tora L.) a mistura que mostrou antagonismo significativo foi $0,96 \mathrm{~kg} / \mathrm{ha}$ de bentazon $+0,10 \mathrm{Kg} /$ /ha de paraquat.

\section{SUMMARY}

EFFECT OF BENTAZON AND PARAQUAT MIXTURES ON WEED CONTROL AND ON BEANS (Phaseolus vulgaris L.)

The present research was carried out to study the selectivity of the mixture bentazon (3-isopropil1H-2,1,3-benzothiadiazin-4(3H)-one 2,2-dioxide) plus paraquat $(1,1$ '-dimethyl-4-4' - bi py ridinium ion) on the bean varieties 'Carioca' and 'Moruna', the effect of the mixture on weed control, and the possible sinergistic effects on weeds. The experiment is being conducted at the experimental area of the Agriculture and Horticulture Department ESALQ/USP - Piracicaba, and at the greenhouse. A randomized complete block design with four replications was used. The evaluation was done by percent of injury to the varieties and weeds, by visual scale. The data were analysed by the formula of Gowing (Colby, 1).

By this method, the injury observed on the varieties was lower less than expected, due to an antagonistic effect. Antagonistic effects were also observed with Digitaria sanguinalis (L.) Scop. The sinergistic effect was observed with Sida glaziovii K. Sch. and Portulacca oleracea mainly with the mixture of $0.48+0.05 \mathrm{~kg}$ a.i./ha of bentazon and paraquat respectively, at six and eight days after application. The mixture of $0.96+0.10$ a.i./ha bentazon + paraquat presented an antagonistic effect for Cassia tora L.

\section{INTRODUÇÃO}

O feijão é uma das principais fontes de proteínas da nossa população. 
Atualmente, devido à escassez de alimentos e com a produção de feijão abaixo das necessidades do mercado interno, a cultura vem tomando impulso, ganhando novas áreas e, com isso, necessitando uma tecnologia mais adequada à produção. O uso de herbicidas na cultura do feijão, apesar de ser prática difundida nos países desenvolvidos, apenas nos últimos anos começou a ser utilizado pelos produtor es brasileiros.

Entre os herbicidas pós-emergentes recomendados para a cultura do feijão está o bentazon $(2,5)$ indicado para o controle seletivo de plantas daninhas de folhas largas. Ao contrário do bentazon, o paraquat não é um herbicida indicado para a cultura do feijão. Esse produto, pode ser utilizado na cultura de soja, apenas em jato dirigido nas entrelinhas da cultura (2), ou então no plantio direto Japós a emergência das plantas daninhas, porém antes da emergência da cultura).

Sinergismo é definido como "a ação cooperativa de diferentes produtos químicos, de modo que o efeito total seja maior do que a soma dos seus efeitos independentes" (6). Kapusta et alii (3) verificaram que a mistura de tanque de bentazon + acifluorfen foi mais eficaz no controle de Ipomoea hederacea em cultura de soja do que cada produto aplicado isoladamente. Putnan e Ries (4) notaram ação sinergística de misturas de herbicidas contendo paraquat no controle de Agropyron repens

(L.) Beauv.

O objetivo da presente pesquisa é estudar o comportamento do bentazon e do paraquat, aplicados isoladamente e em misturas, em área total, sobre a cultura do feijão, a diferentes doses, observando os possiveis efeitos sinergisticos entre esses herbicidas.

\section{MATERIAIS E MÉTODOS}

O experimento está sendo conduzido em condições de campo na área experimental do Departamento de Agricultura e Horticultura da ESALQ em Piracicaba - SP, assim como em casa de vegetação. As cultivares de feijão utilizadas foram 'Moruna' e 'Carioca', ambas com ciclo em torno de 90 dias. 0 delineamento experimental foi o de blocos ao acaso e com quatro repetições. As plantas daninhas existentes no ensaio de campo eram: Digitaria sanguinalis (L.) Scop. (capim-colchão); Sida glaziovii K. Sch. (guanxuma); Ipomoea acuminata Roem et Sch. (corda-de-viola); Portulacca oleracea L. (beldroega) e Cassia tora

L. (fedegoso). A semeadura foi feita no dia $20 / 02 / 81$. Os tratamentos foram aplicados no dia $14 / 03 / 81$, ou seja, 22 dias após o plantio ou 18 dias após a emergência da cultura e das plantas daninhas. No momento da aplicação, a cultura estava no es tádio do $2 .^{\circ}$ ao $3 .^{\circ}$ trifólio e as plantas daninhas com atê $7 \mathrm{~cm}$ de altura, no estádio da $2 .^{a}$ a 4. ${ }^{\text {a }}$ folha verdadeira. Os tratamentos, alêm das testemunhas capinada e sem capina, foram os seguintes: bentazon a 0,48 e $0,96 \mathrm{~kg} / \mathrm{ha}$; paraquat a 0,05 e $0,10 \mathrm{~kg} / \mathrm{ha}$ e misturas de tanque a $0,48+$ $+0,05 ; 0,96+0,10 ; 0,48+0,10$ e $0,96+0,05 \mathrm{~kg} / \mathrm{ha}$ de bentazon + paraquat, respectivamente. O critêrio de avaliação adotado foi o da "percentagem de injúria" às cultivares e às espêcies de plantas daninhas, com avaliações visuais feitas aos 2, 4, 6

e 8 dias após a aplicação dos herbicidas, após o que o experimento foi totalmente capinado, exceto a testemunha sem capina.

Para interpretação dos dados, foi utilizada a fórmula de Gowing citada por Colby (1) que é a seguinte:

\section{$\mathbf{E}=\mathbf{X}+\mathbf{Y}(100-\mathbf{X}) / 100$}

onde: $\mathrm{E}=$ percentagem de injúria esperada pela mistura de bentazon + paraquat às doses $\mathrm{db}+\mathrm{dp}$ respectivamente;

$\mathrm{X}=$ percentagem de injúria observada pelo bentazon à dose $\mathrm{db}$.

$\mathrm{Y}=$ percentagem de injúria observada pelo paraquat à dose dp.

Se a injúria observada na planta for maior que a esperada, a combinação ê sinergística $(+1$; se for menor é antagonistica (-) se for igual ê aditiva (0). Nos quadros 1,2 e 3 os valores da injúria esperada (E) estão entre parênteses.

A cultura não foi levada atê a colheita, devido à seca juntamente com a alta temperatura que a mesma sofreu durante o seu ciclo. Alêm disso, o local apresentou pesada infestação de capimcolchão $(70 \%$ da área coberta pelas plantas daninhas, havendo aproximadamente 350 plantas $/ \mathrm{m}^{\prime}$ das espécies citadas no momento da aplicação), o que prejudicou sensivelmente a cultura no início do seu ciclo vegetativo. Novos experimentos estão sendo conduzidos em condições de campo e casa de vegetação para se ter melhores observações do comportamento das misturas em diferentes condições ambientais.

\section{RESULTADOS E DISCUSSÃO}

O antagonismo ficou bastante caracterizado em ambas as cultivares de feijão (quadro 1). Aplicado isoladamente, o bentazon foi totalmente seletivo às cultivares, nas duas doses ensai- 
Quadro 1. Efeitos sinergísticos (+), antagonisticos (-) e aditivos (0) entre misturas de diferentes doses de bentazon e paraquat, dados em \% de injúria $n$ as cultivares de feijão 'Carioca' e 'Moru na'. - ESALQ - 1981.

\begin{tabular}{|c|c|c|c|c|c|c|c|}
\hline \multirow{3}{*}{$\begin{array}{l}\text { Dias após } \\
\text { aplicaçio }\end{array}$} & \multirow{3}{*}{$\begin{array}{c}\text { Bentazon } \\
\mathrm{kg} / \mathrm{ha}\end{array}$} & \multicolumn{3}{|c|}{ CARIOCA } & \multicolumn{3}{|c|}{ MORUNA } \\
\hline & & \multirow[b]{2}{*}{$\mathbf{0}$} & \multicolumn{5}{|c|}{ Paraquat - kg/ha } \\
\hline & & & 0,05 & 0,10 & $\mathbf{0}$ & 0,05 & 0,10 \\
\hline 2 & $\begin{array}{l}0 \\
0,48 \\
0,96\end{array}$ & $\begin{array}{l}0 \\
0\end{array}$ & $\begin{array}{l}21 \\
6(21)-15 \\
6(21)-15\end{array}$ & $\begin{array}{r}34 \\
11(34)-23 \\
6(34)-28\end{array}$ & $\begin{array}{l}0 \\
0\end{array}$ & $\begin{array}{l}29 \\
7(29)-22 \\
7(29)-22\end{array}$ & $\begin{array}{r}43 \\
13(43)-30 \\
7(43)-36\end{array}$ \\
\hline 4 & $\begin{array}{l}0 \\
0,48 \\
0,96\end{array}$ & $\begin{array}{l}0 \\
0\end{array}$ & $\begin{array}{l}16 \\
3(16)-13 \\
4(16)-12\end{array}$ & $\begin{array}{l}29 \\
8(29)-21 \\
3(29)-26\end{array}$ & $\begin{array}{l}0 \\
0\end{array}$ & $\begin{array}{l}24 \\
4(24)-20 \\
5(24)-19\end{array}$ & $\begin{array}{l}37 \\
9(37)-28 \\
5(37)-32\end{array}$ \\
\hline 6 & $\begin{array}{l}0 \\
0,48 \\
0,96\end{array}$ & $\begin{array}{l}0 \\
0\end{array}$ & $\begin{array}{l}9 \\
0(9)-9 \\
0(9)-9\end{array}$ & $\begin{array}{l}1 \\
3(17)-14 \\
0(17)-17\end{array}$ & $\begin{array}{l}0 \\
0\end{array}$ & $\begin{array}{l}15 \\
0(15)-15 \\
0(15)-15\end{array}$ & $\begin{array}{l}27 \\
5(27)-22 \\
0(27)-27\end{array}$ \\
\hline 8 & $\begin{array}{l}0 \\
0,48 \\
0,96\end{array}$ & $\begin{array}{l}0 \\
0\end{array}$ & $\begin{array}{l}5 \\
0(5)-5 \\
0(5)-5\end{array}$ & $\begin{array}{l}11 \\
0(11)-11 \\
0(11)-11\end{array}$ & $\begin{array}{l}0 \\
0\end{array}$ & $\begin{array}{l}8 \\
0(8)-8 \\
0(8)-8\end{array}$ & $\begin{array}{l}23 \\
0(23)-23 \\
0(23)-23\end{array}$ \\
\hline
\end{tabular}

Quadro 2. Efeitos sinergisticos (+), antagonísticos (-) e aditivos (0) entre misturas de diferentes doses de bentazon eparaquat, dados em \% de injúria, para guanxuma e beldroega ESALQ - 1981

\begin{tabular}{|c|c|c|c|c|c|c|c|}
\hline \multirow{3}{*}{$\begin{array}{l}\text { Dia após } \\
\text { apticaçấo }\end{array}$} & \multirow{3}{*}{$\begin{array}{c}\text { Bentazon } \\
\mathrm{kg} / \mathrm{ha}\end{array}$} & \multicolumn{2}{|c|}{ GUANXUMA } & & \multicolumn{3}{|c|}{ BELDROEGA } \\
\hline & & \multicolumn{6}{|c|}{ Paraquat - kg/ha } \\
\hline & & $\mathbf{0}$ & 0,05 & 0,10 & $\mathbf{0}$ & 0,05 & 0,10 \\
\hline 2 & $\begin{array}{l}0 \\
0,48 \\
0,96\end{array}$ & $\begin{array}{l}16 \\
33\end{array}$ & $\begin{array}{l}59 \\
79(66)+13 \\
76(73)+3\end{array}$ & $\begin{array}{l}75 \\
75(79)-4 \\
85(83)+2\end{array}$ & $\begin{array}{l}33 \\
37\end{array}$ & $\begin{array}{l}66 \\
83(77)+6 \\
74(79)-5\end{array}$ & $\begin{array}{l}76 \\
81(84)-3 \\
85(85) 0\end{array}$ \\
\hline 4 & $\begin{array}{l}0 \\
0,48 \\
0,96\end{array}$ & $\begin{array}{l}27 \\
63\end{array}$ & $\begin{array}{l}53 \\
85(66)+19 \\
87(83)+4\end{array}$ & $\begin{array}{l}75 \\
83(82)+1 \\
91(91) 0\end{array}$ & $\begin{array}{l}33 \\
47\end{array}$ & $\begin{array}{l}60 \\
84(73)+11 \\
75(79)-4\end{array}$ & $\begin{array}{l}76 \\
87(84)+3 \\
89(87)+2\end{array}$ \\
\hline 6 & $\begin{array}{l}0 \\
0,48 \\
0,96\end{array}$ & $\begin{array}{l}43 \\
30\end{array}$ & $\begin{array}{l}44 \\
89(68)+21 \\
93(89)+4\end{array}$ & $\begin{array}{l}55 \\
87(74)+13 \\
94(91)+3\end{array}$ & $\begin{array}{l}37 \\
65\end{array}$ & $\begin{array}{l}50 \\
89(69)+20 \\
87(83)+4\end{array}$ & $\begin{array}{l}65 \\
89(78)+11 \\
93(88)+5\end{array}$ \\
\hline 8 & $\begin{array}{l}0 \\
0,48 \\
0,96\end{array}$ & $\begin{array}{l}55 \\
87\end{array}$ & $\begin{array}{l}23 \\
93(65)+28 \\
98(90)+8\end{array}$ & $\begin{array}{l}35 \\
90(71)+19 \\
99(92)+7\end{array}$ & $\begin{array}{l}46 \\
75\end{array}$ & $\begin{array}{l}43 \\
93(69)+24 \\
93(86)+7\end{array}$ & $\begin{array}{l}55 \\
90(76)+14 \\
99(89)+10\end{array}$ \\
\hline
\end{tabular}


Quadro 3. Efeitos sinergisticos (+), antagonísticos (-) e aditivos (0) entre misturas de diferentes doses de bentazon e paraquat, dados em porcen tagem de injúria, para capim-colcháo, corda-de-viola e fedegoso. - ESALQ - 1981.

\begin{tabular}{|c|c|c|c|c|c|c|c|c|c|c|}
\hline \multirow{3}{*}{$\begin{array}{l}\text { Dias após } \\
\text { aplicaçiòo }\end{array}$} & \multirow{3}{*}{$\begin{array}{c}\text { Bentazon } \\
\mathbf{k g} / \mathbf{h a}\end{array}$} & \multicolumn{3}{|c|}{ CAPIM-COLCH Ão } & \multicolumn{3}{|c|}{ CORDA-DE-VIOLA } & \multicolumn{3}{|c|}{ FEDEGOSO } \\
\hline & & \multirow[b]{2}{*}{0} & & & \multicolumn{3}{|c|}{ Paraquat - kg/ha } & \multirow[b]{2}{*}{0} & \multirow[b]{2}{*}{0,05} & \multirow[b]{2}{*}{0,10} \\
\hline & & & 0,05 & 0,10 & 0 & 0,05 & 0,10 & & & \\
\hline 2 & $\begin{array}{l}0 \\
0,48 \\
0,96\end{array}$ & $\begin{array}{l}0 \\
0\end{array}$ & $\begin{array}{l}56 \\
25(56)-31 \\
34(56)-22\end{array}$ & $\begin{array}{l}70 \\
65(70)-5 \\
45(70)-25\end{array}$ & $\begin{array}{l}49 \\
56\end{array}$ & $\begin{array}{l}69 \\
84(84) 0 \\
75(86)-11\end{array}$ & $\begin{array}{l}76 \\
75(88)-13 \\
93(89)+4\end{array}$ & $\begin{array}{r}0 \\
13\end{array}$ & $\begin{array}{l}35 \\
36(35)+1 \\
33(43)-10\end{array}$ & $\begin{array}{l}60 \\
45(60)-15 \\
43(65)-22\end{array}$ \\
\hline 4 & $\begin{array}{l}0 \\
0,48 \\
0,96\end{array}$ & $\begin{array}{l}0 \\
0\end{array}$ & $\begin{array}{l}36 \\
20(36)-16 \\
24(36)-12\end{array}$ & $\begin{array}{l}69 \\
63(69)-6 \\
35(69)-34\end{array}$ & $\begin{array}{l}53 \\
65\end{array}$ & $\begin{array}{l}64 \\
87(83)+4 \\
83(87)-4\end{array}$ & $\begin{array}{l}77 \\
83(89)-6 \\
93(92)+1\end{array}$ & $\begin{array}{l}0 \\
9\end{array}$ & $\begin{array}{l}25 \\
29(25)+4 \\
27(32)-5\end{array}$ & $\begin{array}{l}56 \\
46(56)-10 \\
36(60)-24\end{array}$ \\
\hline 6 & $\begin{array}{l}0 \\
0,48 \\
0,96\end{array}$ & $\begin{array}{l}0 \\
0\end{array}$ & $\begin{array}{l}26 \\
14(26)-12 \\
15(26)-11\end{array}$ & $\begin{array}{l}60 \\
44(60)-16 \\
29(60)-31\end{array}$ & $\begin{array}{l}60 \\
85\end{array}$ & $\begin{array}{l}51 \\
87(80)+7 \\
91(92)-1\end{array}$ & $\begin{array}{l}73 \\
86(89)-3 \\
97(96)+1\end{array}$ & $\begin{array}{l}0 \\
0\end{array}$ & $\begin{array}{l}16 \\
17(16)+1 \\
21(16)+5\end{array}$ & $\begin{array}{l}41 \\
36(41)-5 \\
24(41)-17\end{array}$ \\
\hline 8 & $\begin{array}{l}0 \\
0,48 \\
0,96\end{array}$ & $\begin{array}{l}0 \\
0\end{array}$ & $\begin{array}{l}16 \\
6(16)-10 \\
9(16)-7\end{array}$ & $\begin{array}{l}49 \\
33(49)-16 \\
17(49)-32\end{array}$ & $\begin{array}{l}60 \\
90\end{array}$ & $\begin{array}{l}40 \\
93(76)+17 \\
97(94)+3\end{array}$ & $\begin{array}{l}56 \\
89(82)+7 \\
98(96)+2\end{array}$ & $\begin{array}{l}0 \\
0\end{array}$ & $\begin{array}{l}13 \\
13(13) 0 \\
11(13)-2\end{array}$ & $\begin{array}{l}33 \\
31(33)-2 \\
21(33)-12\end{array}$ \\
\hline
\end{tabular}


adas. O paraquat, aplicado isoladamente, foi fitotóxico às cultivares. Essa fitotoxicidade foi caracterizada por manchas necróticas na folha, não coalescentes. Apenas as folhas que receberam o produto, entretanto, mantiveram esse sintoma. As folhas novas que iam surgindo não apresentavam sintoma algum de injúria, e, com o passar do tempo, a planta ia se recuperando. A mistura provocou leve clorose até o $8 .^{\circ}$ dia após a aplicação, desaparecendo em seguida.

$\mathrm{O}$ efeito antagonístico também ficou bastante evidente no capim-colchão, em todas as misturas ensaiadas. Nessa espécie, o comportamento da mistura de doses menores foi o inverso do comportamento das doses maiores, ou seja, o efeito antagonístico da primeira ia diminuindo com o passar do tempo, ao passo que o efeito das doses maiores manteve-se semelhante até $0 \quad 8 .^{\circ}$ dia após a aplicação. No fedegoso, o efeito antagonístico foi pronunciado apenas na mistura das doses maiores. As outras combinações não exerceram efeitos antagonísticos ou sinergísticos significativos. Efeito sinergístico foi apenas observado na guanxuma e na beldroega, na mistura de doses menores. Esse efeito foi se acentuando com o passar do tempo até o $8 .^{\circ}$ dia após a aplicação. $\mathrm{Na}$ corda-de-viola os efeitos não foram muito evidentes.

Os sintomas de injúria das misturas nas plantas daninhas foram semelhantes àqueles provocados pelo bentazon aplicado isoladamente, variando de intensidade conforme as doses de cada produto presente nessa mistura. Esses sintomas foram caracterizados por manchas necróticas cinza-escuro, inicialmente nos bordos das folhas e posteriormente coalescendo em manchas maiores, evoluindo pelo interior do limbo foliar. O paraquat, a exemplo do que ocorreu com as cultivares de feijão, provocou nas plantas daninhas manchas necróticas marrom-claro no limbo foliar, não coalescentes e sem evolução.

\section{LITERATURA CITADA}

1. Colby, S.R.. Calculating Synergistic and Antagonistic Responses of Herbicide Combinations. Weeds. Urbana, 15(1):20-22, 1967.

2. Hertwig, $K$. von. Manual de Herbicidas, Desfolhamentes, Dessecantes e Fitorreguladores. Sáo Paulo, Editora Agronơmica Ceres Ltda., p. 54 e 119, 1977

3. Kapusta, G.; Strieker, C.F. e McGinnity, P.J. Ivyleaf morningglory control in soybeans. In: North Central Weed Control Conference, 33.', Proceedings p. 66.

4. Putnan, A.R. \& Ries, S.K.. The Synergistic Action of Herbicide Combinations Contai. ning Paraquat on Agropyron repens (L.) Beauv. Weed Research, Oxford, 7(3):191-199, 1967.

5. Rodrigues, B.N. Controle de Ervas em Cultura de Feijáo. In: Cultura do Feijáo no Estado do Paraná, Londrina, PR. Fundação IAPAR, 18:67-68, 1980 .

6. Weed Science Society of America. Report of the terminology comitee. Weeds, Urbana 12 (4):311-332, 1964. 